\title{
ESTIMULACION DEL REMANENTE VISUAL DE NIÑOS DE BAJA VISION, CON UN PROGRAMA INFORMATICO Y SU EFECTO EN EL RENDIMIENTO ACADEMICO
}

\section{STIMULATION OF THE VISUAL REMAINING OF LOW VISION CHILDREN BY A SOFTWARE PROGRAM AND ITS EFFECT ON THE SCHOOL PERFORMANCE}

\author{
Gloria Anabel Alcívar Pincay \\ gloria.alcivar@uleam.edu.ec \\ Santo Domingo Bravo Loor \\ santos.bravo@uleam.edu.ec \\ Jhonny Saulo Villafuerte Holguin \\ Jhonny.villafuerte@uleam.edu.ec
}

Universidad Eloy Alfaro de Manabí. Facultad de Ciencias de la Educación. Place of Birth, 130802, Manta (Ecuador)

El objetivo de este trabajo es potenciar el aprendizaje escolar de niños y niñas con deficiencia visual, apoyados en la aplicación del programa informático de estimulación visual que fue diseñado por los autores. Este estudio cuasi experimental cuenta con la participación de 30 niños y niñas entre 1 y 6 años de edad. La evaluación del remanente visual se realizó con una valoración clínica y la aplicación de los instrumentos EEV Barraga (1985) y VAP-CAP Blanksby (1993) en pretest y postest. Los resultados reflejan la mejora del rendimiento escolar de los participantes, relacionado directamente al progreso significativo de la función visual.

Palabras clave: Escuelas inclusivas, Eficacia de la escuela, Innovaciones docentes, Discapacidad visual, Tecnología.

The objective of this work is to enhance the school learning of children with visual impairment by the supported through the implementation of a visual stimulation software program, designed by the authors. This quasi-experimental study has the participation of 30 children between 1 to 6 years old. The evaluation of the visual rem anent was performed with a clinical assessment and application of instruments IV Barraga (1985) and VAP-CAP Blanksby (1993) in a pretest and postest. The results reflect improved educational outcomes for participants, directly related to the signification improvement of the children's visual function.

Keywords: Inclusive schools, School effectiveness, teaching innovations, Visually Handicapped, Technology. 


\section{Introducción.}

En la etapa de educación básica, los niños y niñas desarrollan las capacidades funcionales fundamentales para la vida pero, se observan retrasos marcados en el proceso de aprendizaje cuando existe discapacidad visual. Al respecto, Barraga (1985) indica: «cuanto más experiencias visuales tenga un niño, más conductos cerebrales se estimularán, lo que dará lugar a una mayor acumulación de imágenes visuales variadas y de recuerdos» (Barraga, 1985, p.28) pero, no todos los alumnos y alumnas con discapacidad visual son participes de las diversas tareas y experiencias visuales que se ofrecen en el centro escolar; ya que se ha dado el incremento en la diversidad del alumnado, y los docentes no están facilitando la participación de estudiantes con discapacidad dentro de sus aulas (Corral, Bravo \& Villafuerte, 2015). Por su parte Hyvärinen (1988), evidencia que los niños y niñas no desarrollan la visión hasta el máximo potencial por si solos; por lo tanto, hace falta someterlos a estimulación para lograrlo y es conveniente, incentivar el uso de la visión durante la mayor parte del día. En esta misma línea, Alberti y Romero (2010) hace referencia a la discapacidad visual cerebral como el producto de una disfunción del cerebro y no de los ojos, «no pueden transmitir, procesar o integrar de manera correcta la información visual que reciben sus ojos» (p.25), y aun cuando estos se encuentran en óptimas condiciones físicas y funcionales, se producen por una disfunción en la parte posterior de la vía óptica o en alguna de las estructuras cerebrales implicadas en la visión. La estimulación visual interviene cuando se descubre que los niños y niñas presentan déficit en el desarrollo visual, en este caso ellos deben ser motivados a realizar tareas visuales que les enseñe a «ver» ya que con la estimulación visual, lo que buscamos es desarrollar las capacidades físicas, cognitivas y emocionales de niños y niñas para potenciar su desenvolvimiento independiente.

Este trabajo de investigación se suscribe en la tipología cuasi experimental y recurre al uso de las TIC, para incidir en la función visual de un grupo de 30 niños y niñas, quienes reportan baja visión y deficiencias asociadas, clínicamente valoradas. Se trata de un trabajo interdisciplinar que parte de la selección del grupo intervención, la determinación de la función visual al inicio y al final del proyecto, la ejecución de una intervención educativa que se apoya en TIC; cuyos resultados indican que es posible mejorar la función visual de manera exitosa en la niñez.

\subsection{La discapacidad y la estimulación visual en la niñez.}

Desde la perspectiva educativa Barraga (1985, p.28) define a la discapacidad visual como la «alteración en la estructura o funcionamiento en el órgano de la visión [...] la cual, interfiere con el aprendizaje que se puede lograr a través del sentido de la vista»; Por su parte Faye (1972) citado por Bueno (1972) define a la baja visión como el «tramo intermedio entre la visión normal y la ausencia total o casi total de visión, caracterizado por un sistema visual con alteraciones irreversibles [...] que constituye un obstáculo para el desarrollo de la vida de las personas» (Bueno, 1972, p.3).

En cuanto al desarrollo visual del menor, se conoce que los niños y niñas nacen con un desarrollo visual limitado o incompleto. Como lo menciona Hyvärinen (1988), la visión es una función que se va aprendiendo y 
puede mejorar a medida que se la usa. A partir del estudio de Frebel (2007) sobre la incidencia de la discapacidad visual cerebral, se ratifica que ésta es más frecuente en niños y niñas con múltiples deficiencias al igual que Black (1980, p. 487) quien afirma que «entre el 40-75\% de los niños y niñas que padecen de parálisis cerebral, tienen también alguna forma de problemas o incapacidades de la vista». La eficiencia visual es valorada en la medida en que las «tareas visuales» son o no efectuadas por la persona; el tiempo que toma la persona para realizarlas, y el grado de dificultad que tiene una tarea para su ejecución, en contraste con la capacidad de independencia funcional que tiene la persona; estas han sido categorizadas en: «discapacidad visual profunda, discapacidad visual severa, y discapacidad visual moderada» Barraga (1997, p. 32).

Está comprobado que la función del sistema visual guarda estrecha relación con otros sistemas corporales, y las personas aprenden a funcionar visualmente creando una relación entre el aprendizaje, el interés y la motivación ya que las oportunidades de lograr un funcionamiento visual óptimo se incrementan cuando el resto visual es utilizado y estimulado de forma constante, respetando factores que pueden ejercer influencia positiva o negativa en los niños y niñas, y en su interés por aprender a «ver». Para Álvarez (2010) la estimulación visual supone una estimulación al cerebro, el cual acumula imágenes visuales y las deposita en la memoria por lo cual, a través de la estimulación se puede lograr que las diferentes funciones visuales se activen, respetando siempre las condiciones individuales y proporcionando un ambiente agradable y seguro para el aprendizaje; y que los niños con discapacidad visual se esfuercen para ver más. Álvarez (2010: p.27) recalca que «a medida que se realiza la estimulación visual, se observará el nivel de resolución en las diferentes tareas, el tiempo que emplee, la facilidad y comodidad con la que lo logre (eficiencia Visual)».

\subsection{Programas educativos y la motivación para el aprendizaje en la niñez con discapacidad visual.}

Los autores concuerdan con que la condición de discapacidad visual concurrente a otras discapacidades, interfieren en aprendizaje de niños y niñas, en la medida en que esta condición reduce la capacidad para utilizar la visión funcional, pudiendo afectar la independencia de la persona (Black, 1988; Cabero, 2001; Frebel 2007). Por otro lado, los programas educativos están diseñados para alcanzar competencias acordes al currículo, edad y nivel educativo donde el sentido de la vista permite a los niños y niñas interactuar con el mundo que les rodea y obtener información sobre tamaño, distancia, formas y colores de los elementos observados; cada objeto o acción observada despierta su interés por «ver y conquistar ese mundo». Para los niños y niñas con deficiencia visual, la meta clave es lograr su independencia funcional, alcanzar autonomía en la ejecución de las actividades cotidianas, elegir y tomar decisiones, desarrollar habilidades adaptativas que les permitirán desenvolverse en distintos ambientes, desempeñar diferentes roles, cubrir sus necesidades básicas, entablar relaciones interpersonales, ser personas independientes y productivas, ocupando un lugar digno en la sociedad a la que pertenece.

Para los estudiantes con deficiencia visual, las adaptaciones curriculares serán el acceso 
a los elementos del currículo, tomando por ejemplo el uso del Sistema Braille, el uso de materiales concretos o adaptados, o el uso de ayudas ópticas que les facilite el desarrollo de tareas visuales, entre otras. Martínez (2009) afirma que «el uso de metodologías y recursos que favorezcan el aprendizaje de los alumnos, también ha sido una práctica dentro de este panorama innovador» (Martínez, 2009, p.3). Su objetivo es facilitar el esfuerzo intelectual necesario para comprender y retener nuevos conocimientos». Los alumnos alcanzarán mayor grado de independencia a medida que logren dar significado a lo que «ven»(Percepción Visual), es decir que, procesan y comprenden la información que reciben para lo cual, requieren del uso de herramientas didácticas, prótesis u otros mecanismos de soporte, pero, su permanente utilización demanda de una fuerte carga motivacional. La motivación es concebida por algunos autores como las «fuerzas que actúan sobre una persona o en su interior, y provocan que se comporte de una forma específica, encaminada hacia una meta» Hellriegel \& Slocum (2004, p.126).

Desde otra perspectiva Robbins (2004) define a la motivación como «procesos que dan cuenta de la intensidad, dirección y persistencia del esfuerzo de un individuo por conseguir una meta» (p.3), por lo tanto, la motivación puede ser concebida como una fuerza que siempre encamina a conseguir una meta, al logro de un objetivo, que en el caso de los niños y niñas sería: la satisfacción de sus propias necesidades. Se debe fomentar la motivación por el aprendizaje, proporcionando guía y apoyo temporal o permanente dependiendo de su condición, educarles y entrenarles hacia el logro de ese grado de independencia García (2003). Se procura el respeto de los deseos, y diferencias individuales, las que hacen que cada persona logre adaptarse a las circunstancias de manera distinta según su capacidad cognitiva, grado de discapacidad visual, tiempo desde la aparición de la misma, entorno en el que se desenvuelve, grado de apoyo de la familia, entre otros; siempre considerando el artículo 3 de los derechos fundamentales propuesto en el 2006 durante la Convención sobre los derechos de las personas con discapacidad «el respeto de la dignidad inherente, la autonomía individual, incluida la libertad de tomar las propias decisiones, y la independencia de las personas». (Convención sobre los derechos de las personas con discapacidad, 2006, p.5).

Paralelamente, Hyvärinen (1988) plantea la conveniencia de que el aprendizaje de las habilidades visuales y el uso eficiente del resto visual, se realice en forma de juegos para que los niños y niñas nunca lo vivan como una enseñanza o un entrenamiento formal. El juego es un valioso recurso motivacional para enseñar de forma creativa a un niño, ya que a través del juego se experimenta y logra comprender la realidad que rodea a las personas, y aporta al desarrollo de la imaginación. Se trata de una gran fuente de información que refuerza áreas como la orientación y movilidad, la coordinación viso-manual y el área recreativa.

\subsection{Las TIC y la educación inclusiva.}

Alcantud y Soto (2003) indican que «el uso de las tecnologías como medio para incrementar, mantener o mejorar las capacidades funcionales de los individuos es una práctica común en el ámbito de la intervención con personas con discapacidad» (p. 24). 
Más tarde Sunkel (2006, p.8) define a las TIC como «...herramientas y procesos para acceder, recuperar, guardar, organizar, manipular, producir, intercambiar y presentar información por medios electrónicos...». Desde ambas definiciones, se comprende que dichos procesos permiten el manejo de la información y su transmisión, siendo el computador, tabletas y teléfonos inteligentes los elementos más representativos. Kranzberg, citado por Valle de Frutos (2011) sobre la tecnología sugiere que el impacto de estas en la sociedad depende del uso que las personas le den «La tecnología no es buena ni mala, ni tampoco neutral» (41). Pero es Alba (2006) quien indica que las TIC buscan potenciar las capacidades funcionales de las personas con discapacidad; estas procuran facilitar la comunicación, ayudan a adoptar postura corporal adecuada, y sobre todo, aportan al logro de la movilidad e independencia de personas con discapacidad.

Así mismo, en el manual de asistencia tecnológica de México: SEP (2006) en Longoria (2008) se indica que la Asistencia Tecnológica es el «...equipo o servicio que puede ser usado con el objetivo de suplir, aumentar, mantener o mejorar las capacidades funcionales de las personas con discapacidad» (Longoria, 2008, p 31) de tal manera que las tecnologías proveen de accesibilidad para la vida independiente de las personas con discapacidad en diversas áreas, entre ellas: comunicación, posicionamiento y movilidad, aprendizaje, hogar, trabajo, actividades del diario vivir, juego y recreación, entre otros. Bueno y Espejo (2005) proponen actividades para el desarrollo de habilidades visuales, que se estimulan con ordenador tales como: la enseñanza del movimiento de los ojos, coordinación visomotora, percepción figurafondo, constancia perceptual, percepción de las posiciones de los cuerpos en el espacio, percepción de las relaciones entre los objetos en el espacio, memoria visual, visualización, imaginación, y elaboración mental. Sobre los beneficios de las tecnologías Cabero, Córdoba, et. al (2007) indican que, este dependerá del tipo y grado de discapacidad que presenta el estudiante; ayudan a superar las limitaciones que se derivan de las discapacidades cognitivas, sensoriales, y motrices del alumnado; favorecen la autonomía de los estudiantes; ahorran tiempo para la adquisición de habilidades, capacidades y destrezas en los estudiantes; favorecen el diagnóstico de cada estudiante; respaldan un modelo de comunicación y de formación multi sensorial; propician la formación individualizada; evitan la marginación; facilitan la inserción sociolaboral; proporcionan momentos de ocio; favorecen la disminución del sentido de fracaso académico y personal.

Ridgway, L. y Mckears, S. (1985) en Sacco (2009: 4) afirmaron que «si tienes alguna discapacidad, la computadora puede cambiar tu vida», y proponen un abanico de alternativas para superar dificultades específicas, incrementar las opciones de comunicación, y de inclusión educativa, social, laboral, entre otras. Según Collins (1998), los beneficios de la introducción de las TIC en el sector educación alcanzaron las salas de educación especial, aunque este no fuera el propósito en un principio, los maestros han sido provistos por innovadoras herramientas que apoyan su quehacer y por ende, los estudiantes aprenden con mayor facilidad y entusiasmo; lo que significa un gran aporte al proceso de enseñanza y aprendizaje. Al respecto Chacón (2007) señala 
que si se planifica la introducción de las TIC para la atención a las NEE (Necesidades Educativas Especiales), sin pretender alcanzar la «normalización» se estará provocando un impacto positivo en el individuo con discapacidad, su dignidad y auto consideración; ya que las TIC dinamizan la enseñanza, agilitan las tareas, fomentan: interacción social y trabajo cooperativo, actividad intelectual, iniciativa, creatividad, la toma de decisión, y da opción a elegir, lo que lo que para Cabero (2001) representa, el aportar en la construcción de ciudadanos autónomos, productivos y felices.

Entre los estudios previos dirigidos al desarrollo de la función visual desde el entorno educativo, aparece en la década de los años 1960 el primer programa de estimulación visual por parte de la Dra. Natalie Barraga. Se trata de un programa secuenciado que respeta las etapas de desarrollo de la visión normal. El objetivo es fortalecer la funcionalidad visual de los niños y niñas con baja visión, brindándoles experiencias visuales adaptadas a sus características individuales y entorno Barraga (1975). Otros trabajos basados en la secuencia del desarrollo de las funciones visuales normales fue propuesto por Chapman \& Tobin (1986), denominado el método «Mira y Piensa»; donde se puede apreciar una meticulosa subdivisión de las funciones perceptivo-visuales, basado en el principio de que el resto visual no es solo útil sino que también es educable, y contiene información sobre los recursos, materiales y ambientes usados para incentivar a los niños a usar su visión residual de manera más efectiva.

El estudio de Hyvärinen (1988), evidenció que para que los niños y niñas logren su máximo pontencial visual, ellos deben ser atendidos con estimulación o entrenamiento para lograrlo; y son las horas del día, las más convenientes para incentivar al niño a usar su visión durante el día.

Los estudios de Hammarlund (1994) en el campo de educación especial con énfasis en la discapacidad visual, delimitan las características a nivel de las formas y los colores utilizados para la estimulación visual efectiva y el uso del remanente visual, como aspectos a considerar en nuevas intervenciones educativas de estas características. El objetivo de nuestro estudio es incrementar la independencia funcional en menores con déficit visual a través de la estimulación a través de las TIC.

\section{Metodología.}

Este trabajo de tipo cuasi experimental, en su modalidad cuantitativa identifica los aspectos de significativa relevancia, tales como la influencia de la estimulación visual sobre la función visual; que denotan la complejidad de la educación destinada a personas con discapacidad, cuando ésta es realizada responsablemente y tributa al propósito de aportar a la satisfacción de las necesidades de la vida presente y futura de los niños y niñas con déficit visual asociada a una discapacidad. Se cuenta con una muestra representativa compuesta por 30 estudiantes con baja visión y con discapacidades asociadas que acuden a la Unidad Educativa Especializada «Angélica Flores Zambrano» situada en Manta, Ecuador. Se pretende influir sobre la función visual en el grupo participante, siendo los factores considerados: la capacidad de percepción visual de los participantes; es decir, la capacidad que permite al individuo adquirir conciencia del mundo que lo rodea, 
de sí mismo y de los demás; y la interpretación que cada persona da a los estímulos recibidos, dependiendo de las circunstancias o condiciones en la que éste se encuentre. Se trata de un proceso que se relaciona más con la capacidad de aprendizaje de la persona, que con su condición visual, entendiendo que la percepción visual es la habilidad para interpretar lo que se ve, es comprender y procesar la información que se obtiene a través del sentido de la vista (Barraga, 1985).

Los instrumentos para la valoración del grado de dependencia funcional fueron los siguientes:

EEV Escala de Eficiencia Visual de Natalie Barraga (1975), la que evalúa el funcionamiento de las conductas y respuestas visuales de niños y niñas con déficit visual a partir de la observación del grado de desarrollo de las capacidades perceptivo-visuales. El test comprende 48 ítems divididos en 4 secciones, la sección uno relacionada con la discriminación de formas geométricas, contorno de objetos, intensidad claro-oscuro, tamaño y posición; la sección dos comprende discriminación de tamaño, detalle de objeto y figura abstracta, posición espacial, constancia de contorno de imagen, detalle de esquema y objetos; la sección tres relacionada con encaje visual, perspectiva espacial, discriminación de detalles en objetos y figuras abstractas y la sección cuatro comprende discriminación de tamaño, posición, secuencia y relación de los símbolos de letras y palabras y grupos de símbolos; y se aplica a edades comprendidas entre 1 año hasta los 7 años. Para efecto de este trabajo se aplicó este instrumento a los estudiantes cuya edad funcional superaba los cuatro años.

Test VAC CAP (Evaluación visual y programación - Capacidad, programación y procesamiento), cuyo objetivo es evaluar el funcionamiento visual de niños y niñas con dificultades visuales de 0 a 3 años asociadas o no a otra discapacidad, el que consiste en la evaluación de la capacidad visual, percepción de luz, motilidad ocular, percepción de objeto, atención visual, percepción espacial, coordinación viso motriz, comunicación visual, percepción de representaciones bidimensionales. Este instrumento consta de dos niveles de aplicación; el nivel uno evalúa la baja visión (EBV), para descubrir la capacidad y nivel de atención visual de la persona y comprende 9 ítems; y el nivel dos consta de 10 ítems, los que permiten evaluar el procesamiento visual (EPV), en donde se considera la percepción visual, las habilidades viso-cognitiva y los niveles más complejos de atención visual. Las etapas ejecutadas en esta investigación son:

1. Determinación del funcionamiento visual de cada uno de los participantes del grupo intervención al inicio del proceso: Las variables estudiadas son funcionamiento y eficiencia visual. Este procedimiento pedagógico valorativo se realizó de manera individual a cada participante. Los exámenes aplicados fueron: la escala EEV y el Test VACCAP.

2. Ejecución de la intervención educativa apoyada en TIC: Consistió en la exposición del grupo participante al programa E.V.A.C presentado por Alcívar (2014), con el propósito de estimular desde niveles básico hasta nivel avanzado II el remanente visual del grupo participante. Esta etapa tuvo una duración de 6 meses del calendario. La intervención parte desde los niveles más básicos a los más complejos, permitiendo la repetición, hasta lograr el desarrollo de las actividades propuestas (Souza, 2005). 
3. Determinación del funcionamiento visual de cada uno de los participantes del grupo intervención al final del proceso: La evaluación del grupo participante al inicio del proceso siendo las mismas variables estudiadas al inicio del proceso: funcionamiento y eficiencia visual. Se aplicó los instrumentos la escala EEV y el Test VACCAP.

4. Análisis comparativo del estado de dependencia visual del grupo participante al inicio del proceso con los resultados obtenidos al final del proceso. Los datos fueron analizados en contraste entre los resultados de los test EEV y VAC PAC.

\section{Resultados.}

Para la determinación del funcionamiento visual de cada uno de los participantes del grupo intervención al inicio del proceso, fueron seleccionados del grupo general de estudiantes de la escuela especial «Angélica Flores Zambrano» de Manta-Ecuador aquellos niños y niñas que se encontraran dentro del rango de edades comprendidas entre 0 y 6 años, y a los estudiantes quienes presentaban sordoceguera y retos múltiples que se registran en el grupo de 6 a 8 años. Para la pre selección del grupo de intervención, se ejecutó un proceso de observación de conductas visuales de los estudiantes para lo cual, ellos fueron expuestos a diferentes actividades y espacios escolares. Se ratificó la selección de 30 participantes, con la triangulación del análisis de las fichas de evaluación clínica visual de los estudiantes.

Como se puede apreciar en el Gráfico 1, el mayor grupo compuesto por 13 estudiantes se encuentra en el rango entre 4 a menores de 6 años; mientras que el grupo de menor frecuencia es el correspondiente al rango de edad: menores a 2 años con 4 estudiantes.

En el Gráfico 2 se observa que mientras que la discapacidad o retos múltiples es la más frecuente en el grupo intervención, el autismo es aquella que tiene una menor representación en el grupo de estudiantes participantes, que están distribuidos en cuatro grupos funcionales de visión (véase Tabla 1 y Gráfico 3): baja visión funcional profunda, moderada, leve o alterada e impedimento visual cortical.

Aplicación del Test de VAC PAC - Pre intervención.

Fue aplicado a 19 estudiantes. El mapeo fue determinado por las características y desarrollo de habilidades visuales de cada participante a la fecha de inicio del proceso investigativo.

Los resultados indican (Véase Gráfico 4) que 2 estudiantes se encontraron en el rango de edad visual de 0 a 2 meses que representan el 10\%; 1 estudiante en el rango de 2 a 5 meses que representa el 5\%; 1 estudiante en el rango de 5 a 7 meses que representa el $5 \% ; 3$ estudiantes en el rango de 7 a 9 meses que representan el 16\%; 6 estudiantes en el rango de 9 a 12 meses que representa el 32\% ;3 estudiantes en el rango de 12 a 15 meses que representan el 16\%; 3 estudiantes en el rango de 15 a 18 meses que representan el $16 \%$.

A continuación se comparan el logro de los estudiantes frente a la escala categorial de VAC PAC. Se puede observar en el Gráfico 5 el estado al inicio del estudio y el reto por cumplir según cada categoría.

\section{La Escala de Eficiencia Visual EEV de} Natalie Barraga-Pre intervención

Fue aplicado a 11 estudiantes. Se detectó 2 estudiantes en el rango de edad visual de 0 a 2 meses que representan el 10\%; 1 estudiante en el rango de 2 a 5 meses que representa el 5\%; 1 estudiante en el rango de 7 a 9 meses que representa el 5\%; 3 estudiantes en el 


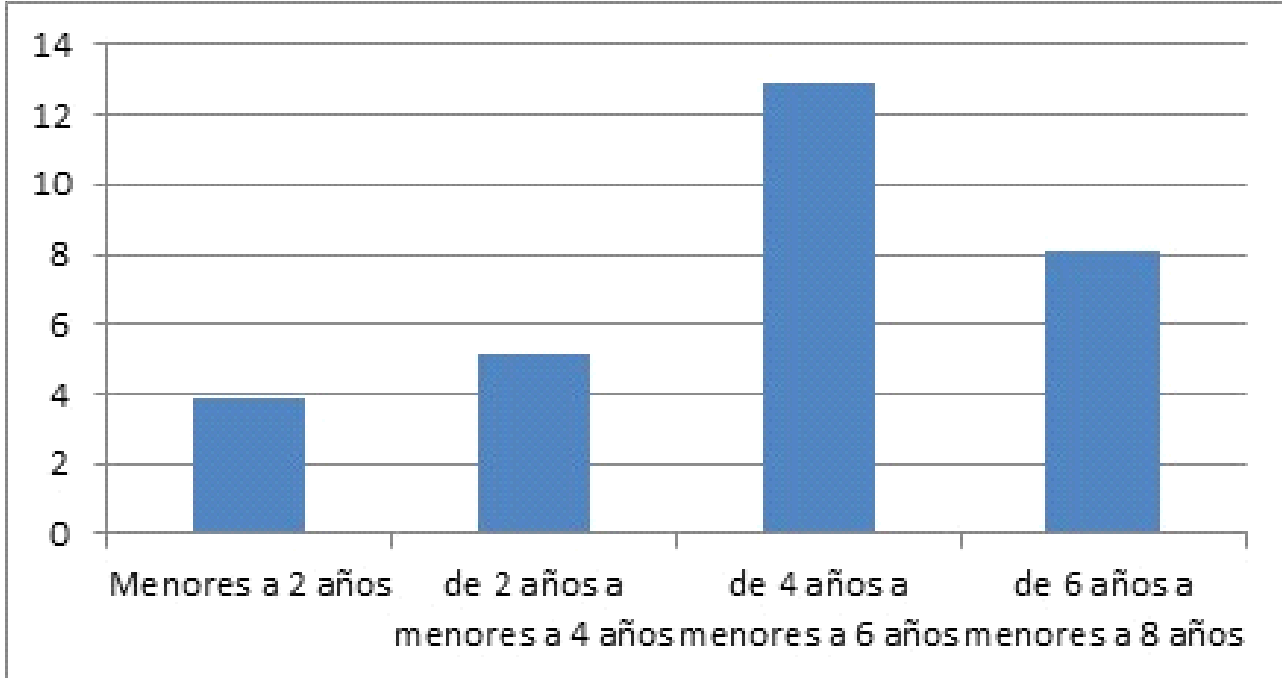

Gráfico 1: Discapacidad concurrente a la baja visión de 30 estudiantes, organizados según edades. Cronológicas en la Escuela Especial «Angélica Flores Zambrano» año lectivo 2014-2015.

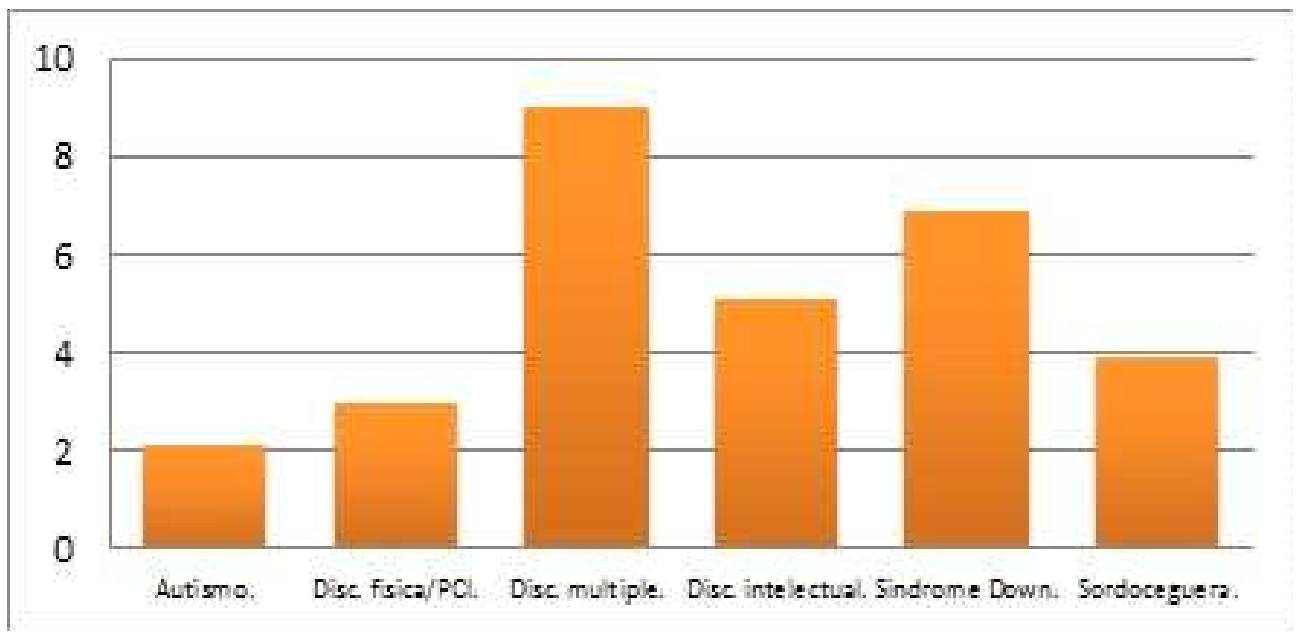

Grafico 2. Tipos de discapacidad observadas en 30 estudiantes de la Escuela Especial «Angélica Flores Zambrano» año lectivo 2014-2015.

Píxel-Bit. Revista de Medios y Educación. $N^{o} 48$ Enero 2016. ISSN: 1133-8482. e-ISSN: 2171-7966. doi: http://dx.doi.org/10.12795/pixelbit.2016.i48.08 


\section{Grados de Visión Funcional}

\begin{tabular}{|l|c|}
\hline Estudiantes con impedimento visual cortical & 5 \\
\hline Estudiantes con baja visión funcional moderado & 9 \\
\hline Estudiantes con visión funcional profunda & 7 \\
\hline Estudiantes con visión funcional leve o alterada & 9 \\
\hline TOTAL & 30 \\
\hline
\end{tabular}

Tabla 1. Categorización del funcionamiento visual de 30 estudiantes con discapacidad visual de la Unidad Educativa Especializada «Angélica Flores Zambrano» año lectivo 2014-2015.

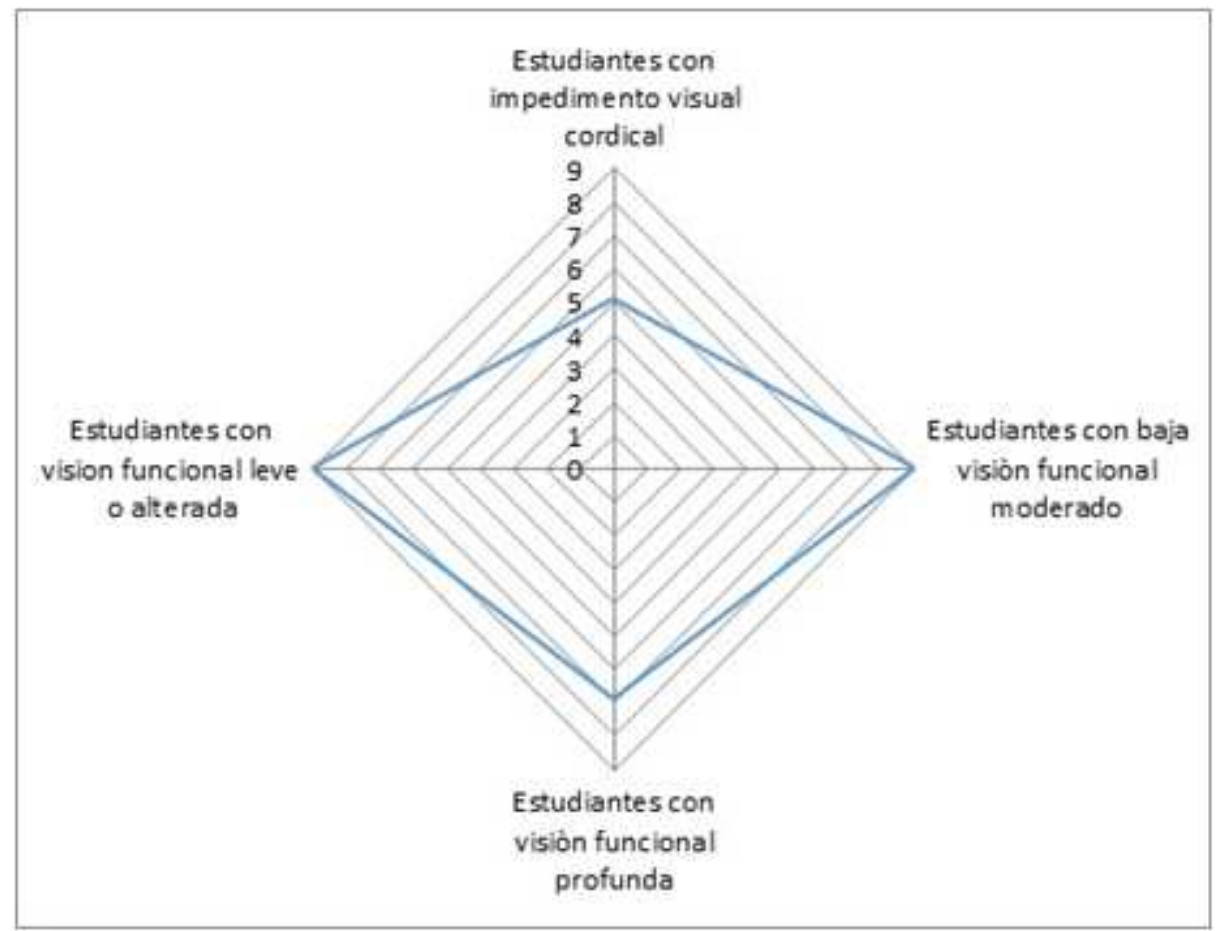

Gráfico 3. Mapeo de funcionamiento visual de 30 estudiantes con discapacidad visual de la Unidad Educativa Especializada «Angélica Flores Zambrano» año lectivo 2014-2015. 


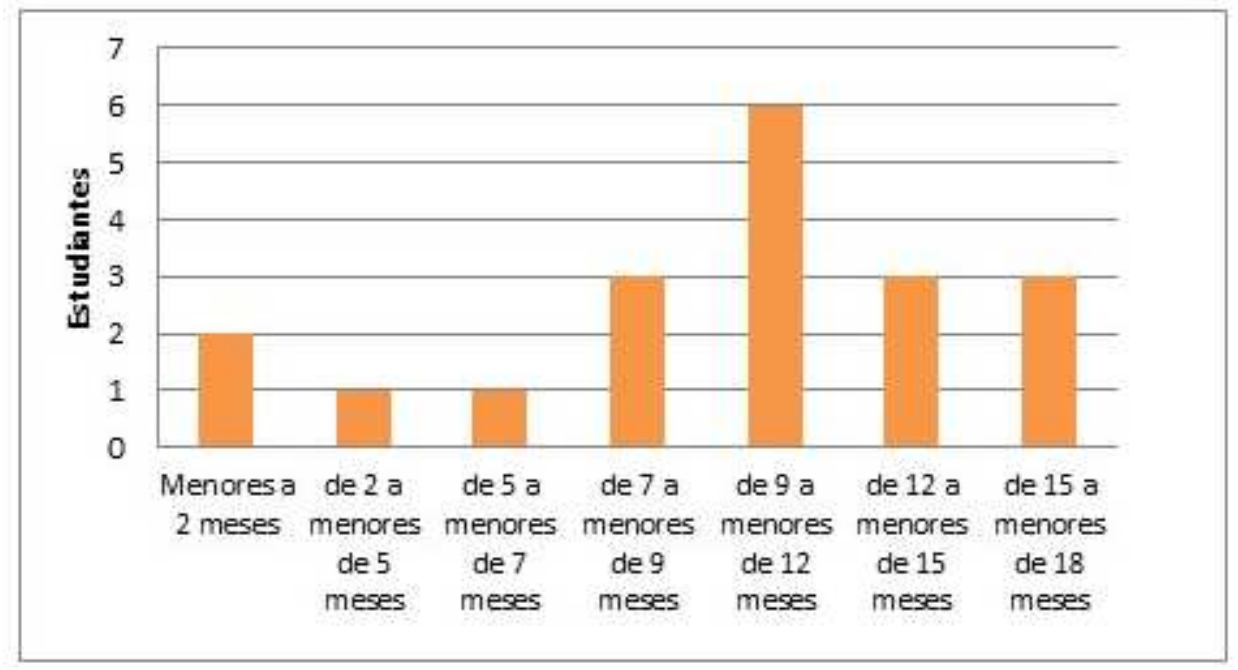

Gráfico 4. Valoración inicial del funcionamiento visual - Test VAP CAP-de 19 estudiantes con discapacidad visual en la Unidad Educativa Especializada "Angélica Flores Zambrano» año lectivo 2014-2015.

de 15 a menores de 18 meses de 12 a menores de 15 meses de 9 a menores de 12 meses de 7 a menores de 9 meses de 5 a menores de 7 meses de 2 a menores de 5 meses Menores a 2 meses

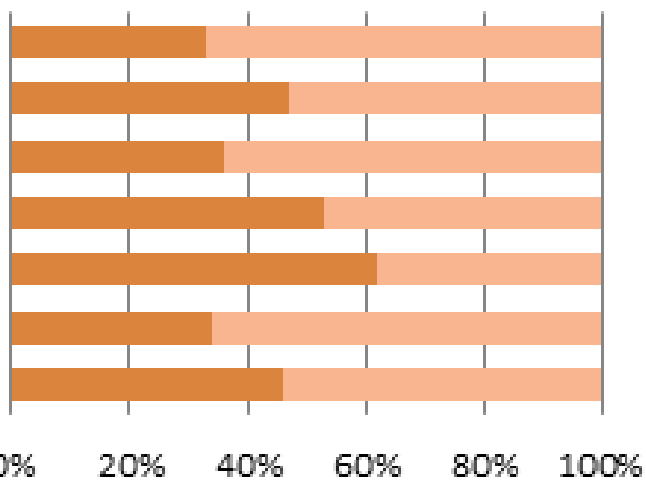
sitiación inicial retos por cumplir

Gráfico 5. Logros iniciales y retos de discapacidad visual de 19 estudiantes en la Unidad Educativa Especializada «Angélica Flores Zambrano» según el test VAC PAC.

Píxel-Bit. Revista de Medios y Educación. No 48 Enero 2016. ISSN: 1133-8482. e-ISSN: 2171-7966. doi: http://dx.doi.org/10.12795/pixelbit.2016.i48.08 


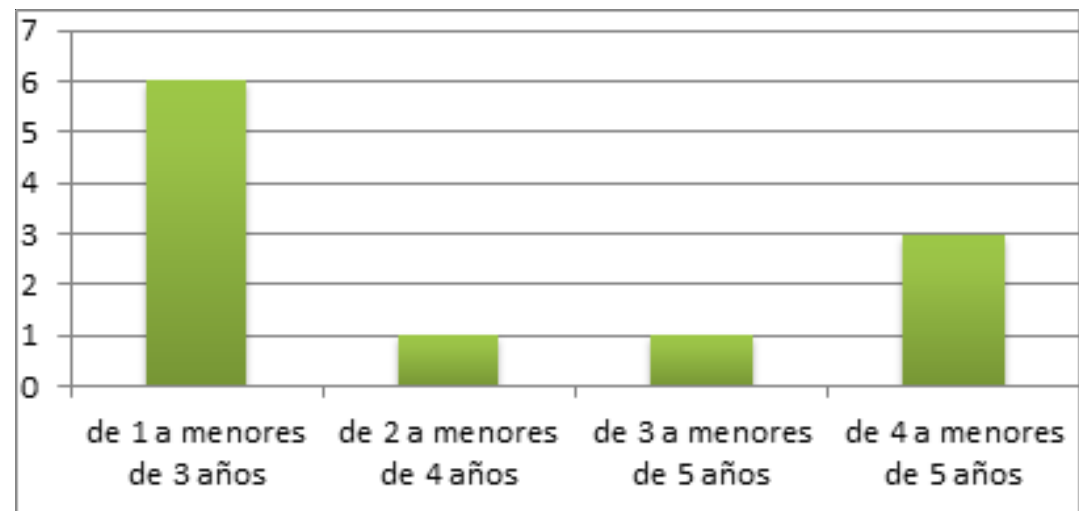

Gráfico 6. Valoración inicial del funcionamiento visual - EEV Barriga -

Unidad Educativa Especializada «Angélica Flores Zambrano», año lectivo 20142015.

rango de 12 a 15 meses que representan el $16 \% ; 3$ estudiantes en el rango de 15 a 18 meses que representan el 16\%; 3 estudiantes en el rango de 30 a 36 meses que representan el 16\%; y 6 estudiantes en el rango de 36 a 48 meses que representan el $32 \%$.

Se observa en el Gráfico 6, que la categoría con mayor frecuencia es la correspondiente a: de 1 a menores de 3 años; mientras que las menores frecuencias se concentran en las categorías: de 2 a menores de 4 años, y de 3 a menores de 5 años.

A continuación en el Gráfico 7 se comparan el logro de los estudiantes frente a la escala categorial de VAC PAC. Se puede observar el estado al inicio del estudio y el reto por cumplir según cada categoría.

Se observa que según los rangos de desarrollo y funcionamiento visual, en comparación de logros alcanzados y retos el

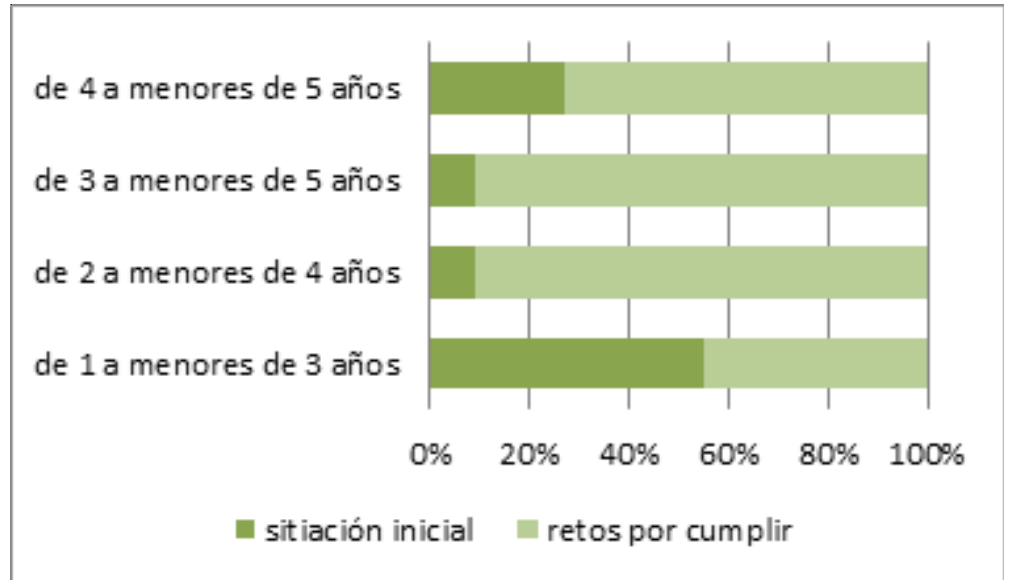

Gráfico 7. Logros iniciales y retos de discapacidad visual de 11 estudiantes en la Unidad Educativa Especializada «Angélica Flores Zambrano» según EEV Barragan. 


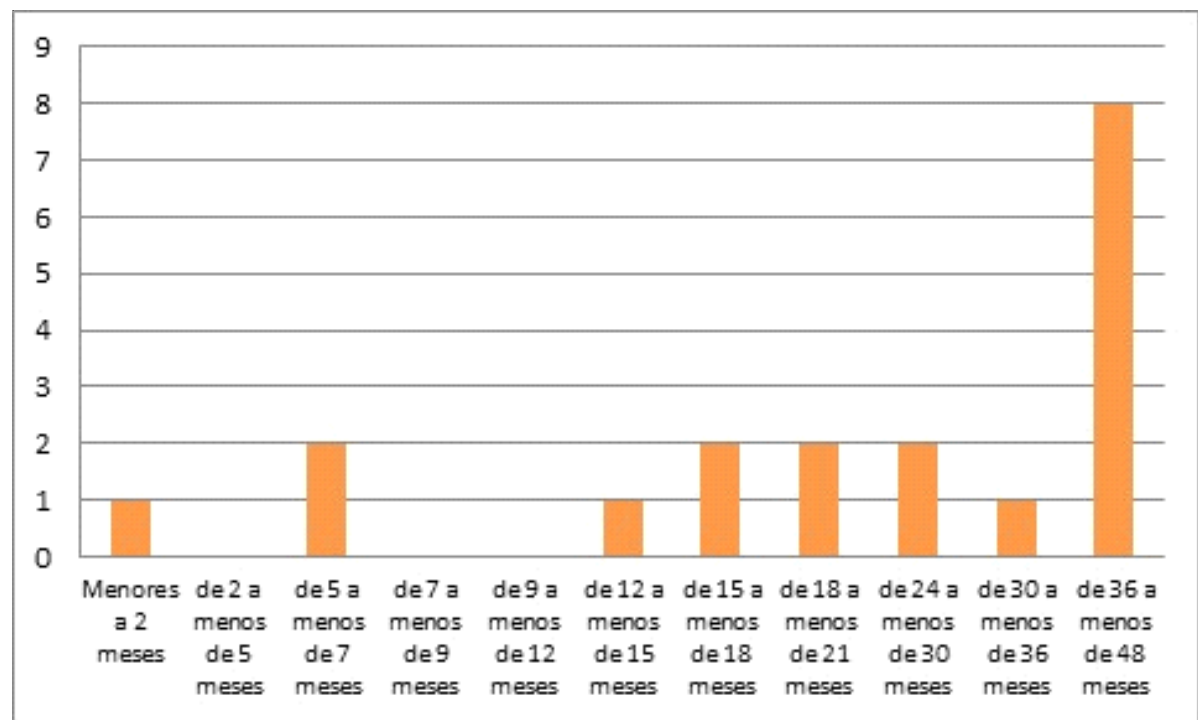

Gráfico 8. Valoración final del funcionamiento visual - Test VAP CAP a estudiantes con discapacidad visual de la Unidad Educativa Especializada «Angélica Flores Zambrano», año lectivo 2014-2015.

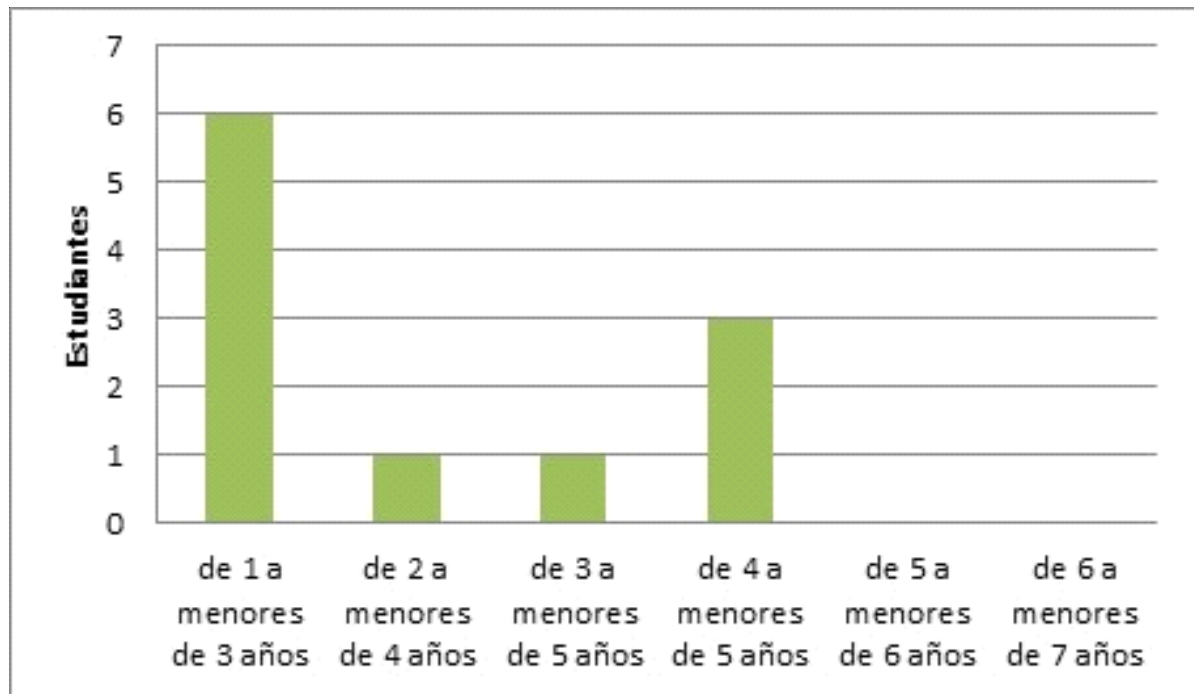

Gráfico 9. Valoración final del funcionamiento visual - EEV Barriga - de estudiantes con discapacidad visual en la Unidad Educativa Especializada "Angélica Flores Zambrano», año lectivo 2014-2015.

Píxel-Bit. Revista de Medios y Educación. No 48 Enero 2016. ISSN: 1133-8482. e-ISSN: 2171-7966. doi: http://dx.doi.org/10.12795/pixelbit.2016.i48.08 


\begin{tabular}{|c|c|c|c|}
\hline $\begin{array}{c}\text { NIVELES VAC PAC DE } \\
\text { FUNCIONAMIENTO } \\
\text { VISUAL }\end{array}$ & $\begin{array}{l}\text { SITUACIÓN } \\
\text { INICIAL }\end{array}$ & $\begin{array}{l}\text { SITUACIÓN } \\
\text { FINAL }\end{array}$ & VARIACIÓN \\
\hline Menores a 2 meses & 2 & 1 & -1 \\
\hline de 2 a menos de 5 meses & 1 & 0 & -1 \\
\hline de 5 a menos de 7 meses & 1 & 2 & 1 \\
\hline de 7 a menos de 9 meses & 3 & 0 & -3 \\
\hline de 9 a menos de 12 meses & 6 & 0 & -6 \\
\hline de 12 a menos de 15 meses & 3 & 1 & -2 \\
\hline de 15 a menos de 18 meses & 3 & 2 & -1 \\
\hline de 18 a menos de 21 meses & 0 & 2 & 2 \\
\hline de 24 a menos de 30 meses & 0 & 2 & 2 \\
\hline de 30 a menos de 36 meses & 0 & 1 & 1 \\
\hline de 36 a menos de 48 meses & 0 & 8 & 8 \\
\hline TOTAL ESTUDIANTES & 19 & 19 & 0 \\
\hline
\end{tabular}

Tabla 2. Comparación de los resultados del Test VAC PAC al inicio y al final del proceso en la Unidad Educativa Especializada «Angélica Flores Zambrano», año lectivo 2014-2015.

rango de 1 a 3 años reportan $56 \%$ de logros; constituyéndose en el avance más relevante.

Análisis complementario de los resultados Test VAC CAP y EEV Barraga - Pre intervención.

Los resultados de la evaluación tanto del test de VAP CAP, al igual que con EEV Escala Barraga, evidencian que el funcionamiento visual del grupo de 30 estudiantes de la
Unidad Educativa Especializada «Angélica Flores Zambrano» de la ciudad de Manta, año lectivo 2014-2015 se ubican por debajo que la edad cronológica de los estudiantes; el nivel de los resultados se relaciona de manera coherente con la edad funcional de los estudiantes; lo cual, es producto de las limitaciones generadas por la discapacidad asociada al déficit visual. 


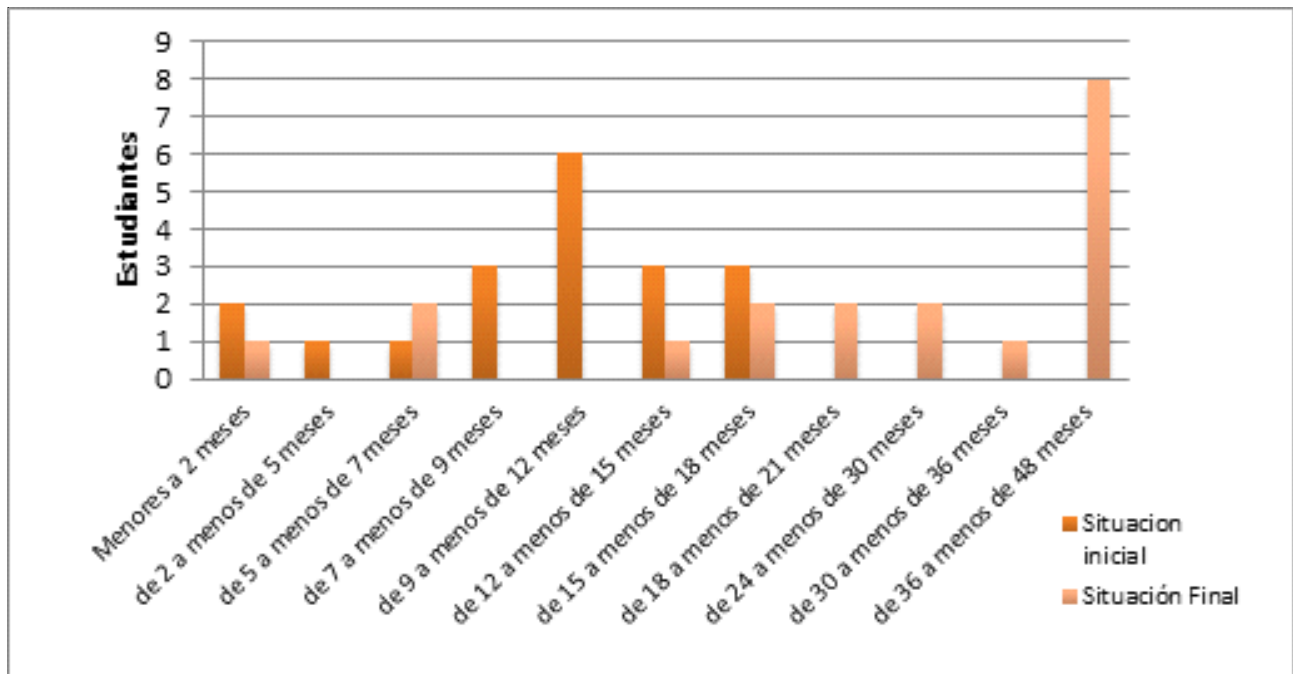

Gráfico 10. Comparación de los resultados del Test VAC PAC aplicados al inicio y al final del proceso en la Unidad Educativa Especializada «Angélica Flores Zambrano», año lectivo 2014-2015.

Determinación del funcionamiento visual de cada uno de los participantes del grupo intervención al final del proceso.

Aplicación Test VAC PAC - Post intervención.

El test VAC PAC fue aplicado al mismo grupo de 19 estudiantes. Los resultados obtenidos posteriores a la intervención, se presentan a continuación en el Gráfico 8 . En la categoría: de 36 a menos de 48 meses se observa la mayor frecuencia alcanzando a 8 casos; mientras que en las categorías: menores a 2 meses, de 12 a menos de 15 meses, y de 30 a menos de 36 meses se reportan 1 caso en cada categoría.

Escala de Eficiencia Visual EEV de Natalie Barraga - Post intervención.

Este post test fue aplicado a los mismos 11 estudiantes del grupo de colaboradores. Se detectó un avance en el funcionamiento visual alcanzando 1 estudiante la categoría: de 6 a menores de 7 años. Los mayores éxitos se observaron en la categoría: de 1 a menores de 3 años, de los cuales 3 estudiantes lograron avanzar una categoría y otros dos estudiantes avanzaron 2 categorías (Véase Gráfico 9).

Análisis comparativo de los resultados de pre-intervención y post-intervención apoyada en el uso de un programa informático:

Los resultados indican que los estudiantes evolucionaron positivamente incrementando su capacidad visual hacia nuevas categorías. El máximo logro lo representan 8 casos quienes alcanzaron el rango: de 36 a menos de 48 meses. Se observó que 1 caso permaneció sin cambios, manteniéndose en el rango: menores a 2 meses (véase Tabla 2 y Gráfico 10).

Se detectó un avance en el funcionamiento visual alcanzando 1 estudiante la categoría: de 6 a menores de 7 años. Los mayores éxitos se observaron en la categoría: de 1 a menores de 3 años, de los cuales 3 estudiantes lograron 


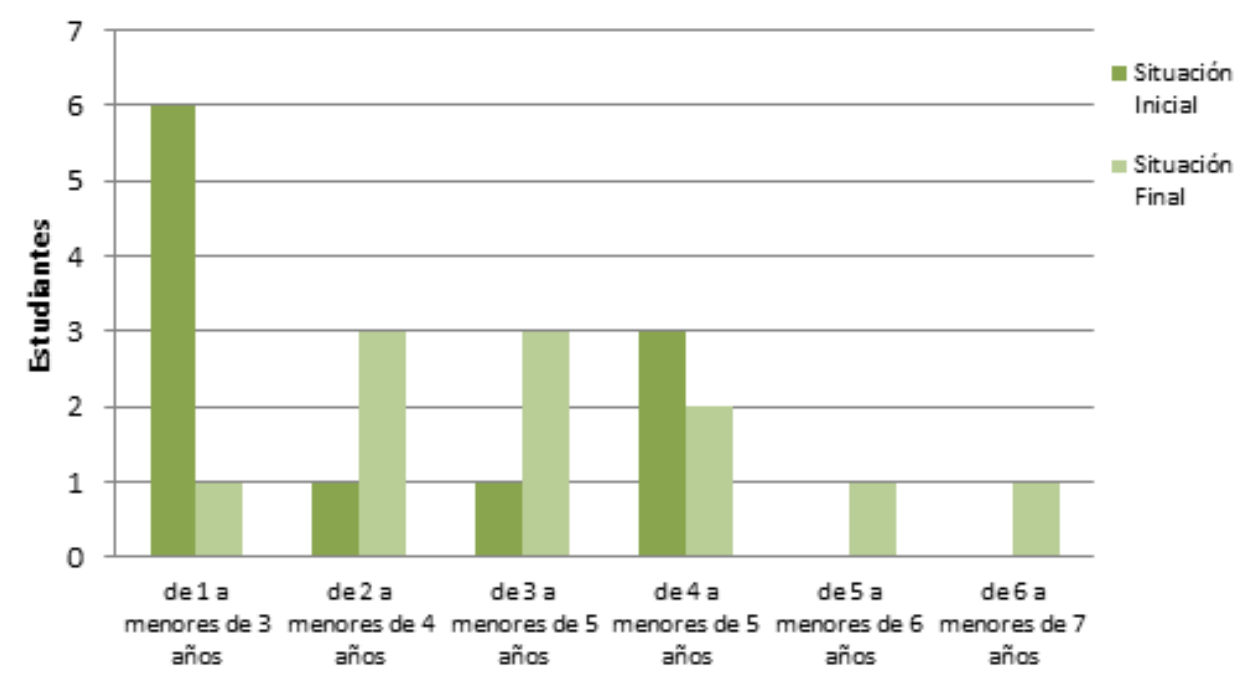

Gráfico 11. Comparación de los resultados del Test VAC PAC aplicados al inicio y al final del proceso en la Unidad Educativa Especializada «Angélica Flores Zambrano», año lectivo 2014-2015.

avanzar una categoría y otros dos estudiantes avanzaron 2 categorías (véase Gráfico 11).

\section{Discusión.}

El remanente visual de niños y niñas con discapacidad visual puede ser ampliado a través de la adecuada estimulación visual, sobre todo si se realiza a través del uso de un computador Buckley \& Brid (1996) y apoyados con los correctivos ópticos adecuados usados desde temprana edad. Los resultados de este trabajo corroboran la posición de Buckley y Brid al confirmar en su parte empírica el mejoramiento del remanente visual de las niñas y niños participantes que han sido expuestos a la ejercitación permanente del remanente visual. Con dicha mejora se logra fortalecer el desarrollo de las habilidades adaptativas en búsqueda de su independencia y autonomía funcional, ya que para adquirir el aprendizaje la vía más factible es la capacidad visual que éste posea; con lo cual, se da por cumplido el objetivo del presente trabajo.

La motivación hacia el aprendizaje y hacia el deseo de «ver» es el elemento imprescindible de las intervenciones basadas en el uso de nuevas tecnologías; las que emplean elementos presentes al entorno del estudiantado y temáticas que resulten de interés para los niños y niñas. Siendo la meta captar y mantener la atención, dedicación y disfrute del estudiantado en el proceso educativo, Hyvärinen (1988) propone que las tareas visuales sean planteadas en forma lúdica, incorporando sonido, movimiento e interactividad para lograr mantener a los niños ejercitando su remanente visual, lo cual es apoyado por los autores 
Con referencia a las ayudas técnicas que fueron tomadas para esta intervención educativa, fue seleccionado el computador que es categorizada por Chacón (2007), como la ayuda electrónica provista de adaptaciones de bajo nivel diseñadas para personas con baja visión, y adaptaciones de alto nivel, comunicadores electrónicas, ayudas para el acceso al computador y a los sistemas informáticos. El computador como herramienta para mediar programas de estimulación visual destinados a estudiantes con baja visión permite generar tareas visuales, bajo una perspectiva globalizadora y coherente con el enfoque ecológico funcional desde el cual, se pone en práctica el currículo.

En lo concerniente a la selección de un programa informático de estimulación del remanente visual en la niñez; este trabajo tomó el E.V.A.C. 2014 que fue diseñado por el equipo investigador pero, puede acudir a materiales que se encuentren en el mercado y su aplicación, para lo cual se deberá adaptar el mismo a las condiciones de la zona próxima de aprendizaje. El proceso deberá ser monitoreado para asegurar se cumpla con las recomendaciones de Hammarlund (1994), es decir que los elementos gráficos deben ser de contornos muy definidos y de un alto contraste aplicando colores primarios. El tiempo de duración de las intervenciones debe ser pactado con el centro escolar y en participación de padres y madres de familia. Los resultados de avance en la función visual, es posible obtenerlos en el $80 \%$ de los casos después de los 3 meses de aplicación de forma disciplinada en sesiones diarias de $45 \mathrm{~min}$.; las cuales deberán estar libres de interrupciones y elementos distractos. Sin embargo, resultados contundentes en el progreso de la función visual se obtendrán después de 6 meses de exposición de los niños y niñas a la herramienta TIC.

Una vez que el niño aprenda a hacer uso de su capacidad visual logrará realizar actividades cotidianas, mejorará su comunicación y relaciones interpersonales, alcanzará independencia en la movilidad, elementos importantes para convivir en sociedad; sin embargo el proceso requiere de un permanente monitoreo, apoyo de parte de profesorado comprometido y movilizado en favor de la educación inclusiva como un compromiso por la eliminación de las barreras y discriminaciones; pero con énfasis en la atención a personas con vulnerabilidad física.

\section{Conclusión.}

1. Los resultados obtenidos en esta investigación ratifican que es posible que niños y niñas con baja visión asociada o no a una discapacidad, mejoren sus niveles de función visual, al ser estimulados con el uso de las TIC, y en concreto el computador y un software adecuado; logrando subir uno o dos niveles en la EEV Bargan y VAC PAC, en periodos de intervención entre 3 y 6 meses.

2. La integración del uso de las TIC en el desarrollo del currículo amplía las oportunidades en el proceso de aprendizaje de los estudiantes con discapacidad, instrumentos como el programa E.V.A.C. y otros softwares disponibles en el mercado enfocados en la estimulación visual, facilitan la adquisición del aprendizaje en ámbitos de las ciencias naturales y entorno. No se dispone al momento de resultados en campos de las matemáticas.

3. El estudiantado con discapacidad visual debe ser permanentemente motivado para aprender y sobre todo para tener el deseo de «ver», caso contrario el potencial que 
constituye realizar actividades agradables con el computador, podrían convertirse en un tiempo que no ayudaría en el desarrollo visual, ni en el aprendizaje de los niños y niñas que tienen esta discapacidad.

\section{Referencias Bibliográficas.}

Alba, C. (2006). Una educación sin barreras tecnológicas. TIC y Educación inclusiva. En J.M. Sancho (Coord.), Tecnologías para transformar la educación (pp. 169-198). Madrid: AKAL.

Alberti, M. \& Romero, O. (2010). Alumnado con discapacidad visual ( ${ }^{\mathrm{a}} \mathrm{Ed}$.). Barcelona: Grao.

Alcantud, M. \& Soto, F. (2003). Tecnologías de ayuda en personas con trastornos de comunicación. Valencia: Nau Libres.

Alcívar, A. (2014). Diseño e implementación de un software de estimulación visual para potenciar la independencia funcional, en niños y niñas de 0 a 6 años con baja visión asociada a otra discapacidad (Tesis de maestría en Educación Especial con mención en Educación de las Personas con Discapacidad Visual). Universidad Politécnica Salesiana Sede Guayaquil.

Álvarez, J. A. (2010). Intervención educativa en alumnos con deficiencia visual. Temas para la Educación. Revista Digital para Profesionales de la Enseñanza, 6. Recuperado de http:// w w w. f e a n d a 1 u c i a . c c o o.e s / docuipdf.aspx?d=6692\&s=

Barraga, N. (1975). Guía del Maestro para el desarrollo de la capacidad del aprendizaje visual y la utilización de la pobre visión. Argentina: AFOB.
Barraga, N. (1985). Disminuidos visuales y aprendizaje. Enfoque evolutivo. Madrid: ONCE.

Barraga, N. (1997). Programa para desarrollar la eficiencia en el funcionamiento visual. En N. Barraga, Textos reunidos (2 ${ }^{\mathrm{a}} \mathrm{ed}$.). Madrid: Organización Nacional de Ciegos Españoles.

Black, P. (1980). Defectos oculares en los niños con parálisis cerebral. British Medical Journal, 281, 487-488.

Blanksby, D. (1993). Evaluación visual y programación. Manual VAPCAP. Córdoba (Argentina): Christoffel Blindemission.

Buckley, S. \& Brid, G. (2006). La educación de las personas con síndrome Down. Madrid: CEPE.

Bueno, M. (1972). Definiciones y clasificaciones en torno a la discapacidad visual. La baja visión y la ceguera. Recuperado en http:// www.juntadeandalucia.es/averroes/caidv/ $\mathrm{i} \mathrm{n} \mathrm{t}$ e $\mathrm{r}$ e d v i s u a $1 / \mathrm{f} \mathrm{t} \mathrm{p}_{-} \mathrm{p}_{-} /$ def_bajavision_ceguera.pdf

Bueno, M. \& Espejo, B. (2005). Actividades básicas de estimulación por ordenador para niños y niñas con escasas respuestas visuales. En III Congreso Virtual Interedvisual sobre autonomía personal con sordoceguera y deficiencia visual. Málaga.

Cabero, J. (2001). Tecnología educativa. Diseño y utilización de los medios en la enseñanza. Barcelona: Paidós.

Cabero, J., Córdoba, M. \& Fernández. J. (2007) (Coords). Las TIC para la igualdad. Nuevas Tecnologías y atención a la diversidad. Sevilla: Eduforma.

Chacón, M. (2007). La atención a la diversidad con medios tecnológicos didácticos. Convención sobre los derechos de las personas con discapacidad, aprobada por la Asamblea General de las Naciones 
Unidas, el 13 de diciembre de 2006, ratificada por España el 30.03.2007.

Chapman, E. \& Ttobin, M. (1986) (Coord.). Mira y piensa. Madrid: ONCE.

Convención sobre los derechos de las personas con discapacidad. (2006). Artículo 3. Principios Generales, p. 5, recuperado en http://www.un.org/esa/socdev/enable/ documents/tccconvs.pdf

Collins, A. (1998). El potencial de las tecnologías de la información para la educación. En C. Vizcarro \& J. León (Ed.), Nuevas Tecnologías para el Aprendizaje. Madrid: Pirámide.

Corral, K., Bravo, S. \& Villafuerte, J. (2015). Situación de la Educación Inclusiva en el cantón Manta al año 2014. Runachay. Revista de Educación.

Faye, E. (1972). El enfermo con déficit visual. Experiencia clínica en adultos y niños. Barcelona: Científico-Médica.

Florez, J. (1995). Patología cerebral en el síndrome de Down: aprendizaje y conducta. En J. Perera (Dr.), Síndrome de Down, aspectos específicos (pp. 27-52). Barcelona: Masson.

Frebel, H. (2007). ¡¿DVC?!, Cómo definirla y qué terminología emplear: deficiencia visual cerebral, cortical o cognitiva. Entre dos Mundos. Revista de Traducción sobre Discapacidad Visual, 33, 77-80.

García, J. (2003). El Movimiento de Vida Independiente Experiencias Internacionales. Madrid: Fundación Luis Vives.

Hammarlund, J. (1994). Los programas de ordenador para niños en edad preescolar visualmente impared. En A.C. Kooijman et al. (eds.), Baja Visión. La investigación y los nuevos avances en la rehabilitación (pp. 338344). Amsterdam: IOS Press.
Hellriegel, D. \& Slocum, J. (2004). Comportamiento organizacional $\left(10^{\mathrm{a}} \mathrm{Ed}\right)$. México: Thomson Learning.

Hyvärinen, L. (1988). La visión normal y anormal en los niños. Madrid: ONCE.

Longoria, M. (2008). El uso de las tics en la asesoría técnica de educación especial en el estado de Chihuahua (México) como estrategia de mejora y optimización del servicio, recuperado en http://gredos.usal.es/ j s pui/bitstream/10366/18454/1/ DDOMI_Uso\%20TICs\%20asesoria\%20 tecnica\%20educacion\%20especial.pdf

Martínez, M. J. (2009). El portafolio para el aprendizaje y la evaluación. Murcia: Editum.

Ridgway, L. \& Mckears, S. (1985). Ayuda del ordenador para personas con discapacidad. Londres: Souvenir Press.

Robbins, S. (2004). Comportamiento Organizacional (10 ${ }^{\mathrm{a}}$ Ed). México: Pearson Educación.

Sacco, A. (2009). Estrategias para la utilización de tecnología en educación especial. Trabajo final integrador presentado para obtener el grado de Especialista en Tecnología Informática aplicada en Educación. Buenos Aires. Disponible en http:/ /postgrado.info.unlp.edu.ar/Carreras/ E s p e c i a 1 i z a c i o n e s / Tecnologia_Informatica_Aplicada_en_Educacion/ Trabajos_Finales/Antonio_Sacco.pdf

Sánchez, M.; López, M.; Martínez, S.; Mirete, A. y García, F. (2009). El uso del portafolios y web-didáctica en educación superior, recuperado en http://webs.um.es/ fags/docs/2011aidipe_portafolio.pdf

SEP (2006). Asistencia Tecnológica. En Todos en la Misma Escuela Asistencia Tecnológica, Secretaría de Educación Pública. Disponible en: http:// 
normalista.ilce.edu.mx/normalista /boletín.

Consultado: 12 de agosto 2014.

Souza, A. (2005). Desarrollo de un ambiente de aprendizaje de carácter cooperativo destinado al alumno con deficiencia intelectual: el software «Hércules y Jiló». Anales del XVII Congreso Internacional de Informática Educativa 2005, Madrid, UnedEspaña.

Sunkel, G. (2006). Las tecnologías de la información y la comunicación (TIC) en la educación en América Latina. Una exploración de indicadores. Santiago: CEPAL

Valle, S. (2011). Cibercultura y civilización universal. Hacia un nuevo orden cultural. Barcelona: Erasmus Ediciones.

Fecha de recepción: 01-10-2015

Fecha de evaluación: 16-10-2015

Fecha de aceptación: 30-10-2015 